\title{
Mechanisms, Then and Now: From Metaphysics to Practice
}

\author{
Stathis Psillos \& Stavros Ioannidis \\ Dept of History and Philosophy of Science \\ University of Athens \\ University Campus \\ 15771 Athens, \\ Greece \\ psillos@phs.uoa.gr \\ sioannidis@phs.uoa.gr
}

\begin{abstract}
For many old and new mechanists, Mechanism is both a metaphysical position and a thesis about scientific methodology. In this paper we discuss the relation between the metaphysics of mechanisms and the role of mechanical explanation in the practice of science, by presenting and comparing the key tenets of Old and New Mechanism. First, by focusing on the case of gravity, we show how the metaphysics of Old Mechanism constrained scientific explanation, and discuss Newton's critique of Old Mechanism. Second, we examine the current mechanistic metaphysics, arguing that it is not warranted by the use of the concept of mechanism in scientific practice, and motivate a thin conception of mechanism (the truly minimal view), according to which mechanisms are causal pathways for a certain effect or phenomenon. Finally, we draw analogies between Newton's critique of Old Mechanism and our thesis that the metaphysical commitments of New Mechanism are not necessary in order to illuminate scientific practice.
\end{abstract}

\section{Introduction}

The mechanical worldview of the seventeenth century was both a metaphysical thesis and a scientific theory. It was a metaphysical thesis insofar as it was committed to a reductionist account of all worldly phenomena to configurations of matter in motion subject to laws. In particular, it was committed to the view that all macroscopic phenomena are caused by, and hence are accounted for, the interactions of invisible microscopic material corpuscles. Margaret Wilson captured this view succinctly:

The mechanism characteristic of the new science of the seventeenth century may be briefly characterised as follows: Mechanists held that all macroscopic bodily phenomena result from the motions and impacts of submicroscopic particles, or corpuscles, each of which can be fully characterised in terms of a strictly limited range of (primary) properties: size, shape, motion and, perhaps, solidity and impenetrability $(1999, x i i i-x i v)$.

But this metaphysical thesis did, at the same time, license a scientific theory of the world, viz., a certain conception of scientific explanation and of theory-construction. To offer a scientific explanation of a worldly phenomenon $\mathrm{X}$ was to provide a configuration $Y$ of matter in motion, subject to laws, such that $Y$ could cause X. A mechanical explanation then was (a species of) causal 
explanation: to explain that $\mathrm{Y}$ causes $\mathrm{X}$ was tantamount to constructing a mechanical model of how Y brings about X. The model was mechanical insofar as it was based on resources licensed by the metaphysical worldview, viz., action of particles by contact in virtue of their primary qualities and subject to laws of motion. ${ }^{1}$

Nearly four centuries later, the mechanical worldview has become prominent again within philosophy of science. It's become known as 'the New Mechanical Philosophy' and has similar aspirations as the old one. New Mechanism, as Stuart Glennan puts it,

\begin{abstract}
says of nature that most or all the phenomena found in nature depend on mechanismscollections of entities whose activities and interactions, suitably organized, are responsible for these phenomena. It says of science that its chief business is the construction of models that describe, predict, and explain these mechanism-dependent phenomena $(2017,1)$.
\end{abstract}

So, New Mechanism too is both a view about science and about the metaphysics of nature. And yet, in New Mechanism the primary focus has been on scientific practice, and in particular on the use of mechanisms in discovery, reasoning and representation (cf. Glennan 2017, 12). The focus on the metaphysics of mechanisms has emerged as an attempt to draw conclusions about the ontic signature of the world starting from the concept of mechanism as it is used in the sciences. According to Glennan, as the research into the use of mechanism in science developed, "it has been clear to many participants in the discussion that metaphysical questions are unavoidable" $(2017,12)$. It is fair to say that New Mechanism aims to ground the metaphysics of mechanisms on the practice of mechanical explanation in the sciences.

The chief aim of this paper is to discuss the relation between the metaphysics of mechanisms and the role of mechanical explanation in the practice of science. It will do that by presenting and comparing the key tenets of Old and New Mechanism. Section 2 will be devoted to the seventeenth century Mechanism. It will present the basic contours of the mechanistic metaphysics and show how it constrained scientific explanation, focusing on the case of gravity. In this section, we will also discuss Isaac Newton's critique of mechanism and highlight the significance of his key thought, viz., that causal explanation should identify the causes and the laws that govern their action, irrespective of whether or not these causes can be taken to satisfy certain (mostly metaphysically driven) constraints, such as being modelled in terms of configurations of matter in motion. Section 3 will focus on the current mechanistic metaphysics and show that it is not warranted by the use of the concept of mechanism in scientific practice. It will show that the currently popular minimal general characterisation of a mechanism is still metaphysically inflated in various ways and will motivate a thin conception of mechanism, which is not committed to any views about the ontological signature of mechanism. This thin conception-what we call 'truly minimal mechanism'-

\footnotetext{
${ }^{1}$ For the purposes of this paper, we ignore issues of mind-body causation and we focus on bodybody causation. We also ignore divisions among mechanists concerning the nature of corpuscles, the existence of vacuum etc.
} 
takes it that mechanisms are causal pathways for a certain effect or phenomenon. Finally, in section 4 we will draw analogies between Newton's critique of Old Mechanism and our critique of New Mechanism. Briefly put, the point will be that causal explanation in the sciences is legitimate even if we bracket the issue of "what mechanisms or causes are as things in the world" (Glennan 2017, 12); or the issue of what activities are and how they are related to powers and the like. The metaphysical commitments of New Mechanism are not necessary in order to illuminate scientific practice.

\section{Old Mechanism: from Metaphysics to Practice}

A rather typical example of the interplay between the metaphysical worldview and the scientific conception of the world in the seventeenth century was the attempted mechanical explanation of gravity.

\subsection{Mechanical Models of Gravity}

Let us start with René Descartes. The central aim of the $3^{\text {rd }}$ and $4^{\text {th }}$ part of Descartes's Principia Philosophiae, published in 1644, was the construction of an account of natural phenomena. In Cartesian physics, the possible empirical models of the world are restricted from above by a priori principles which capture the fundamental laws of motion and from below by experience. Between these two levels there are various theoretical hypotheses, which constitute the proper empirical subject-matter of science. These are mechanical hypotheses; they refer to configurations of matter in motion. As Descartes explains in (III, 46) of the Principia, since it is a priori possible that there are countless configurations of matter in motion that can underlie the various natural phenomena, "unaided reason" is not able to figure out the right configuration of matter in motion. Mechanical hypotheses are necessary but experience should be appealed to, in order to pick out the correct one:

[W] are now at liberty to assume anything we please [about the mechanical configuration], provided that everything we shall deduce from it is \{entirely in conformity with experience (III, 46; 1982, 106).

These mechanical hypotheses aim to capture the putative causes of the phenomena under investigation (III, 47). Hence, they are explanatory of the phenomena. Causal explanation-that is, mechanical explanation-proceeds via decomposition. It is a commitment of the mechanical philosophy that the behaviour of observable bodies should be accounted for on the basis of the interactions among their constituent parts and particles; hence, on the basis of unobservable entities. In (IV, 201; 1982, 283), Descartes states that sensible bodies are composed of insensible particles. But to get to know these particles and their properties a bridge principle is necessary; that is, a principle that connects the micro-constituents with the macro-bodies. According to this principle, the properties of the minute particles should be modelled on the properties of macro-bodies. Here is how Descartes put it:

Nor do I think that anyone who is using his reason will be prepared to deny that it is far better to judge of things which occur in tiny bodies (which escape our senses solely because of their smallness) on the model of those which our senses perceive occurring in large bodies, than it is to devise I know not what new things, having no similarity with those things which are observed, in order to give an account of those things [in tiny 
bodies]. [E.g., prime matter, substantial forms, and all that great array of qualities which many are accustomed to assuming; each of which is more difficult to know than the things men claim to explain by their means\} (IV, 201; 1982, 284).

In this passage Descartes does two things. On the one hand, he advances a continuity thesis: it is simpler and consonant with what our senses reveal to us to assume that the properties of micro-objects are the same as the properties of macro-objects. This continuity thesis is primarily methodological. It licenses certain kinds of explanations: those that endow matter in general, and hence the unobservable parts of matter, with the properties of the perceived bits of matter. It therefore licenses as explanatory certain kinds of unobservable configurations of matter; viz., those that resemble perceived configurations of matter. On the other hand, however, Descartes circumscribes mechanical explanation by noting what it excludes; that is by specifying what does not count as a proper scientific explanation. He's explicit that the Aristotelian-scholastic metaphysics of substantial forms and powerful qualities is precisely what is abandoned as explanatory by the mechanical philosophy².

All this was followed in the investigation of the mechanism of gravity and the (in)famous vortex hypothesis according to which the planets are carried by vortices around the sun. A vortex is a specific configuration of matter in motion-matter revolving around a centre. The underlying mechanism of the planetary system then is a system of vortices:

[T] he matter of the heaven, in which the Planets are situated, unceasingly revolves, like a vortex having the Sun as its center, and [...] those of its parts which are close to the Sun move more quickly than those further away; and [...] all the Planets (among which we \{shall from now on\} include the Earth) always remain suspended among the same parts of this heavenly matter (III, 30; 1982, 196).

The very idea of this kind of configuration is suggested by experience, and by means of the bridge principle it is transferred to the subtle matter of the heavens. Hence, invisibility doesn't matter. The bridge principle transfers the explanatory mechanism from visible bodies to invisible bodies. More specifically, the specific continuity thesis used is the motion of "some straws for other light bodies\}... floating in the eddy of a river where the water doubles back on itself and forms a vortex as it swirls" (op.cit.). In this kind of motion we can see that the vortex carries the straws "along and makes them move in circles with it" (op.cit.). We also see that

some of these straws rotate about their own centers, and that those which are closer to the center of the vortex which contains them complete their circle more rapidly than those which are further away from it (op.cit.).

More importantly for the explanation of gravity, we see that

although these whirlpools always attempt a circular motion, they practically never describe perfect circles, but sometimes become too great in width or in length (op.cit.).

\footnotetext{
2 In (IV, 204; 1982, 286) Descartes accepts that scientific explanation does not require the truth of the claims about the microconstituents of things. In the next paragraph, however, he argues that his explanations have 'moral certainty' (IV, 205; 1982, 286-7).
} 
Given the continuity thesis, we can transfer this mechanical model to the motion of the planets and "imagine that all the same things happen to the Planets; and this is all we need to explain all their remaining phenomena" (op.cit.). Notably, the continuity thesis offers a heuristic for discovering plausible mechanical explanations.

Christiaan Huygens (1690) came to doubt the vortex theory "which formerly appeared very likely" to him $(1997,32)$. He didn't thereby abandon the key tenet of mechanical philosophy. For Huygens too the causal explanation of a natural phenomenon had to be mechanical. He said referring to Descartes:

Mr Descartes has recognized, better than those that preceded him, that nothing will be ever understood in physics except what can be made to depend on principles that do not exceed the reach of our spirit, such as those that depend on bodies, deprived of qualities, and their motions $(1997,1-2)$.

Huygens posited a fluid matter that consists of very small parts in rapid motion in all directions and which fills the spherical space that includes all heavenly bodies. Since there is no empty space, this fluid matter is more easily moved in circular motion around the centre, but not all parts of it move in the same direction. As Huygens put it "it is not difficult now to explain how gravity is produced by this motion" $(1997,16)$. When the parts of the fluid matter encounter some bigger bodies, like the planets: "these bodies [the planets] will necessarily be pushed towards the center of motion, since they do not follow the rapid motion of the aforementioned matter" (op.cit.). And he added:

This then is in all likelihood what the gravity of bodies truly consists of: we can say that this is the endeavor that causes the fluid matter, which turns circularly around the center of the Earth in all directions, to move away from the center and to push in its place bodies that do not follow this motion (op.cit).

In fact, Huygens devised an experiment with bits of beeswax to show how this movement towards the centre can take place.

Newton of course challenged all this, and along the lines, the very idea that causal explanation should be mechanical. But before we take a look at his reasons and their importance for the very idea of scientific explanation, we should not fail to see the broader metaphysical grounding of the mechanical project. For, as we noted, in the seventeenth century Mechanism offered the metaphysical foundation of science.

\subsection{Mechanical vs Non-Mechanical Explanation}

The contours of this endeavour are well-known. Matter and motion are the 'ultimate constituents' of nature; or, as Robert Boyle $(1991,20)$ put it, the "two grand and most catholic principles of bodies". Hence, all there is in nature (but clearly not the Cartesian minds) is determined (caused) by the mechanical affections of bodies and the mechanical laws. Here is Boyle again:

[T] he universe being once framed by God, and the laws of motion being settled and all upheld by his incessant concourse and general providence, the phenomena of the world thus constituted are physically produced by the mechanical affections of the parts of 
matter, and what they operate upon one another according to mechanical laws (1991, 139).

The Boylean conception, pretty much like the Cartesian, took it that the new mechanical approach acquired content by excluding the then dominant account of explanation in terms of "real qualities": the scholastics "attribute to them a nature distinct from the modification of the matter they belong to, and in some cases separable from all matter whatsoever" (1991, 15-16). Explanation based on real qualities, which are distinct (and separable) from matter, is not a genuine explanation. They are posited without "searching into the nature of particular qualities and their effects" $(1991,16)$. They offer sui generis explanations: why does snow dazzle the eyes? Because of "a quality of whiteness that is in it, which makes all very white bodies produce the same effect" (1991, 16). But what is whiteness? No further story about its nature is offered, but just that it's a "real entity" inhering in the substance: why do white objects produce this effect rather than that? Because it is in their nature to act thus.

Descartes made this point too when, in his Le Monde, he challenged the scholastic rivals to explain how fire burns wood, if not by the incessant and rapid motion of its minute parts. In his characteristic upfrontness, Descartes contrasted two ways to explain how fire burns wood. The first is the Aristotelian way, according to which 'the 'form' of fire, the 'quality' of heat, and the 'action' of burning" are "very different things in the wood" (Descartes 2004, 6). The other is his own mechanistic way: when the fire burns wood,

it moves the small parts of the wood, separating them from one another, thereby transforming the finer parts into fire, air, and smoke, and leaving the larger parts as ashes $(2004,6)$.

This causal explanation, based as it is on matter in motion, is preferable precisely because it is explanatory of the burning; in contrast, the Aristotelian is not, precisely because it does not make clear the mechanism by which the fire consumes the wood:

[Y] ou can posit 'fire' and 'heat' in the wood and make it burn as much as you please: but if you do not suppose in addition that some of its parts move or are detached from their neighbours then I cannot imagine that it would undergo any alteration or change (2004, $6)$.

To the then dominant account of real qualities the new mechanical metaphysics juxtaposed a different view of qualities. For something to be a quality it should be determined by the mechanical affections of matter, that is, by "virtue of the motion, size, figure, and contrivance, of their own parts" (Boyle $1991,17)$. Hence, there can be no change in qualities unless there is a change in mechanical affections. Though "catholic or universal matter" is common to all bodies (being, as Boyle $(1991,18)$ put it, "a substance extended, divisible, and impenetrable"), it is diversified by motion, which is regulated by laws.

The key point then is that the mechanical account of nature is both a metaphysical grounding of science and a way to do science: offering mechanical explanations of the phenomena. It covers everything, from the very small to the very large. Here is Boyle again: 
For both the mechanical affections of matter are to be found, and the laws of motion take place, not only in the great masses and the middle-sized lumps, but in the smallest fragments of matter; and a lesser portion of it, being as well a body as a greater, must, as necessarily as it, have its determinate bulk and figure $(1991,143)$.

The metaphysical grounding of mechanical explanation renders it a distinct kind of explanation, which separates it sharply from rival accounts. Concomitantly, it becomes very clear what counts as a non-mechanical alternative. An explanation couched in terms of "nature, substantial forms, real qualities, and the like" is "unmechanical" $(1991,142)$. But a sui generis chemical account of nature is unmechanical too. As Boyle put it:

[T] hough chemical explications be sometimes the most obvious and ready, yet they are not the most fundamental and satisfactory: for the chemical ingredient itself, whether sulphur or any other, must owe its nature and other qualities to the union of insensible particles in a convenient size, shape, motion or rest, and contexture, all which are but mechanical affections of convening corpuscles $(1991,147)$.

The opposition to both of these non-mechanical accounts is weaved around a certain metaphysical account of the world as fundamentally mechanical and a reductive-decompositional account of scientific explanation itself.

\subsection{Newton against Mechanism}

When Newton offered a non-mechanical account of gravity, he primarily challenged the idea that legitimate scientific explanation ought to be mechanistic. There is a sense in which Newton prioritised explanation by unification under laws and not by mechanisms. This is seen in the Preface to the second (1713) edition of the Principia, authored by Roger Cotes under the supervision of Newton. In this preface, Cotes presents Newton's method as a midway (a via media) between Aristotelianism and Mechanism. To be sure, mechanical explanations were an improvement over the Scholastic explanations because they relied on demonstrations on the basis of laws. Still, taking "the foundation of their speculations from hypotheses", the mechanists are "merely putting together a romance [i.e. fiction], elegant perhaps and charming, but nevertheless a romance" (Newton 2004, 43).

Thus put, the point sounds epistemic; it concerns the increased risk involved in hypothesising a mechanism which is supposed to underpin, and hence to causally explain, a certain phenomenon. Cotes adds:

But when they [the mechanists] take the liberty of imagining that the unknown shapes and sizes of the particles are whatever they please, and of assuming their uncertain positions and motions, and even further of feigning certain occult fluids that permeate the pores of bodies very freely, since they are endowed with an omnipotent subtlety and are acted on by occult motions: when they do this, they are drifting off into dreams, ignoring the true constitution of things, which is obviously to be sought in vain from false conjectures, when it can scarcely be found out even by the most certain observations (Newton 2004, 43).

Still, it's fair to say that Newton's via media was based on a different understanding of scientific explanation: it should look for causes-hence, scientific explanation should be causal — but the sought after causes need not be mechanical. Newton's way, Cotes says, is to "hold that the causes of all things are 
to be derived from the simplest possible principles", but unlike the mechanists's way, it "assume(s) nothing as a principle that has not yet been thoroughly proved from phenomena". The "explication of the system of the world most successfully deduced from the theory of gravity" is the "most illustrious" example of Newton's way $(2004,32)$.

Newton emphatically denied feigning any hypotheses about the cause of gravity. For him,

it is enough that gravity really exists and acts according to the laws that we have set forth and is sufficient to explain all the motions of the heavenly bodies and of our sea $(2004,92)$.

\section{Gravity according to Newton is a non-mechanical force since it}

operates not according to the quantity of the surfaces of the particles upon which it acts (as mechanical causes used to do), but according to the quantity of the solid matter which they contain, and propagates its virtue on all sides to immense distances, decreasing always as the inverse square of the distances (op.cit.).

He added that the very motion of the comets makes it plausible to think that the regular elliptical motion of the planets (as well as of their satellites) cannot "have their origin in mechanical causes" $(2004,90)$.

In his already mentioned Discourse on the Cause of Gravity (1690), Huygens expressed his dissatisfaction with Newton's failure to offer a mechanical explanation of the cause of gravitational attraction. Favouring his own explanation of gravity in terms of the centrifugal force of the subtle and rapidly moving matter that fills the space around the Earth and the other planets, Huygens noted that Newton's theory supposes that gravity is "an inherent quality of corporeal matter". "But", he immediately added, such a hypothesis "would distance us a great deal from mathematical or mechanical principles" (1997, 35).

Yet Huygens had no difficulty in granting that Newton's law of gravity was essentially correct when it comes to accounting for the planetary system. As he put it:

I have nothing against Vis Centripeta, as Mr. Newton calls it, which causes the planets to weigh (or gravitate) toward the Sun, and the Moon toward the Earth, but here I remain in agreement without difficulty because not only do we know through experience that there is such a manner of attraction or impulse in nature, but also that it is explained by the laws of motion, as we have seen in what I wrote above on gravity $(1997,31)$.

Explaining the fact that gravity depends on the masses and diminishes with distance "in inverse proportion to the squares of the distances from the centre" $(1997,37)$, were, for Huygens, clear achievements of Newton's theory despite the fact that the mechanical cause of gravity remained unidentified.

Commitment to mechanical explanation was honoured by Gottfried Wilhelm Leibniz too. In a piece titled "Against Barbaric Physics: Toward a Philosophy of What There Actually Is and Against the Revival of the Qualities of the Scholastics and Chimerical Intelligences" (written between 1710 and 1716), he defended the mechanical view by arguing that corporeal forces should be grounded mechanically when it comes to their application to the natural world. 
Leibniz was very clear that though he allowed "magnetic, elastic, and other sorts of forces", they are permissible "only insofar as we understand that they are not primitive or incapable of being explained, but arise from motions and shapes" (Leibniz 1989, 313). So, forces are necessary, but a condition for their applicability to the natural world is that they are seen as "arising from motions and shapes". What he took it to be "barbarism in physics" was to posit sui generis, that is non-mechanically grounded, "attractive and repulsive" forces that act at a distance $(1989,314-315)$. Newton's gravity was supposed to be such a barbaric force!

In a letter he sent to Nicolaas Hartsoeker (Hanover, 10 February 1711), Leibniz makes it clear that the proper scientific explanation should be mechanical. It is not enough for scientific explanation to identify the law by means of which a certain force acts; what is also required is the specification of the mechanism by means of which it acts. The mechanism is, clearly, on top of the law and given independently of it. Without the mechanism the power is "an unreasonable occult quality". He says:

Thus the ancients and the moderns, who own that gravity is an occult quality, are in the right, if they mean by it that there is a certain mechanism unknown to them, whereby all bodies tend towards the center of the earth. But if they mean that the thing is performed without any mechanism by a simple primitive quality, or by a law of God, who produces that effect without using any intelligible means, it is an unreasonable occult quality, and so very occult, that it is impossible it should ever be clear, though an angel, or God himself, should undertake to explain it (Newton 2004, 112).

Newton couldn't disagree more. In an unsent letter written circa May 1712 to the editor of the Memoirs of Literature, Newton referred explicitly to Leibniz's letter to Hartsoeker, and stressed that it is not necessary for the introduction of a power-such as gravity - to specify anything other than the law it obeys; no extra requirements should be imposed, and in particular no requirement for a mechanical grounding:

And therefore if any man should say that bodies attract one another by a power whose cause is unknown to us, or by a power seated in the frame of nature by the will of God, or by a power seated in a substance in which bodies move and float without resistance and which has therefore no vis inertiae but acts by other laws than those that are mechanical: I know not why he should be said to introduce miracles and occult qualities and fictions into the world. For Mr. Leibniz himself will scarce say that thinking is mechanical as it must be if to explain it otherwise be to make a miracle, an occult quality, and a fiction (Newton 2004, 116).

Note well Newton's point. The fact that an explanation does not conform to a certain mechanical framework does not make it fictitious, occult or miraculous. Non-mechanical explanations are legitimate insofar as they identify the law that covers or governs a certain phenomenon. Hence, Newton promotes a methodological shift: causal explanation without mechanisms but subject to laws.

Causal explanation then need not be mechanical to be legitimate and adequate. This is Newton's key thought. In breaking with a tradition which brought under the same roof a certain metaphysical conception of the world and a certain view of scientific explanatory practice, Newton distinguished the two and laid emphasis on the explanatory practice itself, thereby freeing it from a 
certain metaphysical grounding.

Though this is not the end of the story of Old Mechanism, ${ }^{3}$ Newton's key thought, we shall argue, is of relevance in the current debates over New Mechanism, to which we shall now turn our attention.

\section{New Mechanism: from Practice to Metaphysics}

It is useful to differentiate between two ways to conceptualise mechanisms in the post-1970 literature. First, mechanism has been used as a primarily metaphysical concept, mostly aiming to illuminate the metaphysics of causation. Second, mechanism has been taken to be a concept used in science, and philosophical accounts of mechanism have aimed to elucidate this concept. To be sure, some philosophical approaches to mechanism, most notably Glennan's (1996), blend these two conceptions (the metaphysical one and the concept-inuse). However, it's fair to say that there are two quite distinct points of origin of the recent philosophical accounts of mechanism: the first starts from metaphysics (as was the case for Descartes and other old mechanists), the second from scientific practice. Using this distinction between mechanism as a primarily metaphysical concept and as a concept-in-use in science, we can differentiate between two kinds of approaches to the metaphysics of mechanisms.

On the first approach, the aim is to show what the connection is between mechanism qua a metaphysical category and other central metaphysical concepts, notably causation. In the context of the metaphysics of causation, 'mechanistic' accounts are theories about the link between cause and effect. Such theories are meant to be anti-Humean in that they view causation as a productive relation, i.e. the cause somehow brings about or produces the effect. The aim of the mechanistic view of causation is to illuminate the productive relation between the cause and the effect by positing a mechanism that connects them, and by explicating 'mechanism' in a suitable way such that causal sequences are differentiated from non-causal ones. The central thought, then, is that A causes B if and only if there is a mechanism connecting A and B.

Two kinds of views have become prominent: those that characterise the mechanism that links cause and effect in terms of the persistence, transference or possession of a conserved quantity (Mackie 1974; Salmon 1997; Dowe 2000); and those that connect a mechanistic account to causal production with a powerbased one (see Harré (1970) for an early such view). Despite their differences, these views share in common the claim that mechanisms are the ontological tie that constitutes Hume's 'secret connexion'. In our (2018), we have called such mechanisms, mechanisms-of. Mechanisms-of are ontological items that underlie or constitute certain kind of processes, i.e. those that can be deemed causal.

Since we have dealt with these accounts in some detail elsewhere (Psillos 2002; Ioannidis \& Psillos 2018), we shall not discuss them further here.

On the second approach, working out a metaphysics of mechanisms is not the starting point but rather the end point of inquiry. Starting with mechanism as a concept-in-use in science, one tries first to give a general characterisation of

\footnotetext{
${ }^{3}$ For more on the development of Old Mechanism, see Psillos (2011).
} 
this concept and then to derive metaphysical conclusions, i.e. conclusions about the (mechanistic) structure of the world.

This kind of bottom-up inquiry has yielded several well-known general accounts of mechanisms as well as theses about the ontic signature of a mechanistic world.

\subsection{The Metaphysics of New Mechanism}

Here are three well-known general characterisations of a mechanism in recent mechanistic literature:

\footnotetext{
Mechanisms are entities and activities organized such that they are productive of regular changes from start or set-up to finish or termination conditions (Machamer, Darden, \& Craver 2000,3).

A mechanism for a behavior is a complex system that produces that behavior by the interaction of a number of parts, where the interactions between parts can be characterized by direct, invariant, change-relating generalizations (Glennan 2002, S344).

A mechanism is a structure performing a function in virtue of its component parts, component operations, and their organization. The orchestrated functioning of the mechanism is responsible for one or more phenomena (Bechtel \& Abrahamsen 2005, 423).
}

The focus on mechanism as a concept-in-use is common to all three accounts; none of the three accounts can be viewed as falling under the rubric of mechanistic theories of causation. And yet, all these and similar accounts yield specific metaphysical commitments about what kind of things in the world mechanisms are. All these accounts are committed to the thesis that a general characterisation of mechanism must itself be cashed out in metaphysical terms. Hence, talk of mechanisms in science is taken to have quite direct consequences about the kind of ontology presupposed by such talk. In order to substantiate this point, let us look at the three accounts mentioned earlier in some more detail.

Peter Machamer, Lindley Darden and Carl Craver's (MDC) account is perhaps the most ontologically inflated, as it is explicitly committed to both entities and activities as distinct and separate ontological categories. It is thus committed to a particular view about the metaphysics of causation: causation within mechanisms is to be characterised in terms of production, where the productive relation is captured by the various different kinds of activities identified by science.

Glennan's case is interesting, since in his (2002) he refrains from taking mechanisms to entail a productive account of causation. Instead, withinmechanism interactions are characterised in terms of invariant, change-relating generalisations. As we will see below, however, Glennan has presently connected his account of mechanisms with a power-based understanding of causation. Hence, he is committed to causal powers as parts of the building blocks of mechanisms.

Lastly, William Bechtel \& Adele Abrahamsen's account does not include a specific characterisation of what mechanistic causation amounts to at all. Here, however, as in the other two accounts, we have a series of general terms the meaning of which needs to be unpacked. So, MDC include in their accounts 
'entities' and 'organisation'; Glennan includes 'complex system' and 'parts'; and Bechtel \& Abrahamsen talk about 'structure', 'function', 'parts' and 'organisation'.

All these accounts suggest the further need to explain what this 'new mechanical ontology' of entities, activities, organisation of parts into wholes etc. amounts to: what, in general terms, the constituents of mechanisms are and what are their relations with more traditional metaphysical categories, such as things, properties, powers and processes.

Notably, there has been a tendency recently to offer a more minimal general characterisation of a mechanism. For example, according to Phyllis Illari \& Jon Williamson:

A mechanism for a phenomenon consists of entities and activities organized in such a way that they are responsible for the phenomenon $(2012,120)$.

Glennan's recent version is almost identical:

A mechanism for a phenomenon consists of entities (or parts) whose activities and interactions are organised so as to be responsible for the phenomenon $(2017,17)$.

Glennan calls this account Minimal Mechanism. The key motivation here is for a general characterisation of mechanism broad enough to capture examples of mechanisms in different fields, from physics to the social sciences. But even in this minimal mode, mechanisms, according to Glennan, "constitute the causal structure of the world" (Glennan 2017, 18).

This minimal account of mechanism might appear to fit the bill of capturing a concept-in-use in science. On closer inspection, however, it is committed to a rather rich metaphysical account of mechanism: the minimal account is not more minimal than the metaphysically inflated accounts noted above. The reason is that both of the foregoing minimal accounts still invite questions about the ontic status of mechanisms. For example: how exactly do entities and activities differ? What is the relation between activities and interactions? How should organisation be understood? Glennan $(2017,13)$ explicitly talks about a "new mechanical ontology" as the upshot of the minimal account. The "minimal mechanism", he adds, "is an ontological characterization of what mechanisms are as things in the world" $(2017,19)$.

New Mechanism, then, aims to provide a new ontology of mechanisms. We can identify three commonly accepted key theses concerning mechanistic ontology:

(1) The world consists of mechanisms.

Thesis 1 is a typical view among mechanists: mechanisms are taken to be things in the world, with objective boundaries. Ours is a mechanistic world. As Glennan puts it at the end of his $(2017,240)$, "[t]hat is just how we have found the world to be".

(2) A mechanism consists of objects of diverse kinds and sizes structured in such a way that, in virtue of their properties and capacities, engage in a variety of different kinds of activities and interactions such that a certain phenomenon $P$ is brought about. 
Thesis 2 (or something very similar) can be taken as the common core of the general characterisation of mechanism as a concept-in-use given by new mechanists. It identifies the components of a mechanism and the relations among them. As mechanisms are things in the world (thesis 1), their components are also particular things in the world. Besides, these parts engage in activities by being "active, at least potentially" (Glennan 2017, 21). Activity is understood as a manifestation of the powers things have. Glennan is quite explicit that "Activities manifest the powers (capacities) of the entities involved in the Activity" $(2017,31)$. Positing powers is supposed to explain why "activities are powerful"; being powerful, activities are what "an entity does, not merely something that happens to an entity" $(2017,32)$. But activities are not enough. Interactions are needed too because "there is no production without interaction" $(2017,22)$. "The fundamental point of ontological agreement among the New Mechanists", as Glennan (2017, 21 n.6) puts it, is that that entities cannot exist without activities or activities without entities. It's not hard to see that the minimal account of mechanism is taken to imply or suggest a rather substantive metaphysical conception of mechanism, which, until further notice at least, is broadly neo-Aristotelian.

(3) To explain a certain phenomenon $\mathrm{P}$ is to offer the mechanism that produces it.

Thesis 3 connects the previous theses with a claim about explanation (and more specifically, causal explanation): since in a mechanistic world phenomena are produced by mechanisms, scientific explanation has to identify the mechanism that produces a certain phenomenon; that is (by thesis 2) to identify the organised entities and activities that produce the phenomenon.

Despite their differences, there are important similarities between Old and New Mechanism (which justify viewing both positions as mechanistic). On the one hand, as we saw, new mechanists differ from their seventeenth century predecessors in that they do not start their analysis with a metaphysical concept of mechanism; rather, they aim at giving a general characterisation of mechanism as a central concept of scientific practice. This characterisation is non-reductive in that it is not committed to the view that mechanisms are configurations of matter in motion subject to laws (and contact action). But, on the other hand, they are committed to mechanisms being configurations of powerful entities engaged in activities and interactions. As Glennan puts it: "Mechanisms are particular and compound, made up of parts (entities) whose activities and interactions are located in particular regions of space and time" $(2017,57)$. Hence, New Mechanism is similar to seventeenth century Mechanism, in that it is committed to a mechanistic ontology. This ontology (theses $1 \& 2$ above), while not a global metaphysics in the sense of the seventeenth century, is still a thesis about the ontic signature of the world. Here is Glennan again:

New Mechanist ontology is an ontology of compound systems. It suggests that the properties and activities of things must be explained by reference to the activities and organization of their parts $(2017,57)$. 
Instead of resulting in a 'flat' ontology where everything there is consists in matter in motion, this new mechanical metaphysics ends up with a hierarchy of particular things-mechanisms-which may contain a diverse set of entities and activities, rather than the limited set endorsed by the corpuscularians, and whose productivity is grounded in causal powers, rather than in a few fundamental laws of motion.

But we can ask: are these ontological commitments really necessary in order to understand scientific practice? Are they licenced by the practice of science? Remember here that the primary aim of new mechanists is to give a general characterisation of mechanism as a concept-in-use. So, ideally, the general account of mechanism should capture as far as possible the extension of a concept-in-use in the various sciences. The minimal account of mechanism discussed so far, though broad enough to play this role, inflates the concept-inuse by making it amenable to a certain metaphysical description of its basic components.

Note that our claim is not that the metaphysical questions are not philosophically interesting questions to ask; they are, especially if we are interested in giving an account of the ontological structure of reality. Moreover, such a kind of project has to be informed by what science has to say about the world. If, however, our aim is to understand how a specific conceptmechanism -is used in scientific practice, these questions seem, at least prima facie, irrelevant, especially if a general characterisation of mechanism is possible that does not include such things.

\subsection{Mechanism in Scientific Practice}

A metaphysically deflationary view of mechanism as a concept-in-use that is broad enough to capture all examples of mechanisms that we find in science seems indeed possible. In our (2017) we have argued that there is an even more minimal account of mechanism than those offered by Illari \& Williamson and Glennan, which captures this concept-in-use. This is achieved by dropping the reference to activities and interactions and by understanding mechanism as the causal pathway of a certain phenomenon, described in the language of theory. According to this account that we call Truly Minimal Mechanism [TMM], a mechanism in science just is a causal pathway described in theoretical language:

TMM: mechanism = causal pathway, described in theoretical language

The central idea behind TMM is that when scientists talk about a 'mechanism', what they try to capture is the way (i.e. the causal pathway) a certain result is produced. Say, for example, that we want to find out how a certain disease state is brought about. What we must look for is a specific mechanism, i.e. a causal pathway that involves various causal links between, for example, a virus and changes in properties of the organism that ultimately lead to the disease state. In pathology, such causal pathways constitute the pathogenesis of a disease, and when pathologists talk about the mechanisms of a disease, it is such pathways that they have in mind (cf. Lakhani et al. 2009; see also Gillies 2017, for a similar account of mechanisms in medicine as causal pathways).

Mechanisms then and causation are closely related: when two events are causally connected, there is a mechanism (that is, a causal pathway) that 
connects them and accounts for the specific way that the cause brings about the effect. Also, scientists succeed in identifying a mechanism, if they succeed in describing the relevant causal pathway in terms of the theoretical language of the particular scientific field.

In life sciences, this is a typical use of 'mechanism'. In our (2017), we have discussed extensively a particular example, the case of apoptosis, i.e. a particular causal pathway (or mechanism) of cell death. The study of apoptosis (and of mechanisms of programmed and physiological cell death in general) in fact transcends particular biological fields, and has involved cytologists, developmental biologists, pathologists, and molecular biologists among others. Because of its broad role, this case offers a nice test case of the concept of mechanism as it is used in science. Apoptosis is described in various biological disciplines as a 'mechanism' of cell death. The common concept of mechanism at play here, we have argued in (2017), is that a mechanism just is a causal pathway. TMM then is the common denominator of all uses of the concept of mechanism in biology and elsewhere.

To further see the plausibility of this view, consider the relation between the concepts of mechanism and function. In the general accounts mentioned earlier, only Bechtel \& Abrahamsen explicitly refer to the behaviour of a mechanism in terms of its function. But in all accounts, a mechanism is always a mechanism for a phenomenon or behaviour, and this can be taken as an implicit reference to the function of the mechanism, which plays a central role in individuating the mechanism. In other words, there is no mechanism without a function, and the function determines what, among everything that happens within a complex system such as an organism, counts as a mechanism. It is an open issue among new mechanists how this commitment to function should be construed and what its consequences for the metaphysics of mechanisms are (cf. Garson 2018; Craver 2013).

However, if we insist on an account of mechanism broad enough to capture all uses of the concept in science, and given that there are scientific fields where the concept of function is not present (e.g. particle physics, solid state physics, astrophysics, cosmology), an account such as TMM seems preferable. Of course, there are contexts (for example, in molecular biology), where a mechanism is automatically a mechanism for a certain function; e.g. apoptosis is a mechanism for cell death, and it also has a homeostatic function within the organism (see our (2017) for more on this point and its relevance for TMM). But it is not clear that a mechanism of star formation, for example, has star formation as its function, unless one takes function to be what the mechanism produces, i.e. its effect. The point here is that if we want to claim that a mechanism of cell death and a mechanism of star formation are in some sense the same kind of thing (i.e. they are both mechanisms), that is if we want to give a general account of a mechanism as a concept-in-use across various scientific fields, TMM seems the most promising candidate. At the same time, TMM can be easily adapted to capture particular uses of 'mechanism' in various contexts where a specific notion of function is presupposed.

We want to resist the temptation to offer a metaphysically inflated account of the causal pathway, in terms of an explicit specification of its ontological constituents. A key reason for this is that the causal pathway should be described in the theoretical language of a specific scientific field, and not in 
some privileged ontological language or even in ontologically loaded terms. This suggests that the form of the description of the mechanism cannot be decided beforehand and in advance of how the concept of mechanism is used. What counts, each time, as a legitimate description of a causal pathway, is something that has to be decided by scientific practice. Instead of imposing various metaphysical categories as those that constitute a general legitimate description of a mechanism, it should be left to the scientists themselves to decide how best to describe mechanisms using the theoretical language they employ to understand and describe the world. TMM has the consequence that a series of questions that new mechanists have been concerned with need not concern us if our aim is to understand scientific practice. ${ }^{4}$

If we identify a mechanism with a causal pathway, would it be required to make a commitment about what the ontological nature of causation is? This does not seem necessary for understanding the concept-in-use. Scientific practice establishes robust causal connections, which can be used for understanding and manipulation, without necessarily being committed to a single and overarching ontic account of causation. Ultimately, whatever fundamental ontological theory of what causation is one might have (e.g. in terms of causal powers or regularities etc.), the identification of causal relationships is based on theorydescribed difference-making relations; this is what scientists look for when establishing causal relations and causal pathways. In this sense, the causal pathway by means of which a phenomenon $Y$ is brought about by a cause $X$, given that $\mathrm{X}$ initiates a chain of events that leads to $\mathrm{Y}$, is the very network of theory-described difference-making relations among the various intermediaries of $\mathrm{X}$ and $\mathrm{Y}$. It is a further question, and one that is not needed to be answered in order to discover and use causal relations in scientific practice, what the truthmakers of these difference-making relations are. Hence, the point here is that in order to understand what a causal pathway (and hence a mechanism) as a concept-in-use is, and to identify mechanisms, we do not need a theory about the metaphysics of causation: TMM is really, ontologically speaking, a truly minimal view $^{5}$.

TMM is best seen in the context of a thesis that we call, following Joseph Henry Woodger (1929) and Robert Brandon (1984), Methodological Mechanism [MM] (see our (2017) for more on Woodger on MM). In his (1929), Woodger distinguished between two ways in which a certain notion can be employed: a metaphysical or ontological way and a methodological one. The latter is when a notion is used for the purposes of description "independently of its metaphysical interpretation". In this case, Woodger says, the notion "is employed methodologically, i.e. simply for the purpose of investigation" $(1929,31)$. The advantage of this use is that the notion can be used in a certain practice and cast light on it independently of whatever difficulties (and controversies) are raised

\footnotetext{
${ }^{4}$ These questions concern, for example, the components and boundaries of mechanisms (cf. Kaiser 2018), the metaphysics of causation (cf. Matthews \& Tabery 2018), and the mechanistic levels (cf. Povich \& Craver 2018).

${ }^{5}$ There are various other questions that can be raised concerning TMM: for example, do we take pathways to be types or tokens? Here again, we defer to practice. Causal pathways, qua things in the world that produce an effect, are concrete particulars. But what is described theoretically in the language of theory is a type of causal pathway.
} 
by the intricate metaphysical debates concerning what its worldly reference is really like. According to Woodger, taking the methodological standpoint amounts to asking "the methodological mechanist what he has to say in support of his contention that the mechanical explanation is the only one which is admissible in science" $(1929,231)$. Hence, MM is a view about mechanistic explanation and its admissibility, and not about the blueprint of the universe.

Methodological Mechanism, we want to claim, is the view that commitment to mechanism in science is adopting a methodological postulate which licenses looking for the causal pathways for the phenomena of interest. Hence, MM licenses adopting TMM.

MM illuminates practice in a way that ontologically inflated accounts of mechanism do not. It accounts for the centrality of mechanisms in scientific discovery and explanation, since according to it discovering mechanisms (i.e. causal pathways) is the central task of science. At the same time, however, it refrains from imposing on scientific practice ontic constraints that are not licensed by it. According to MM, the mechanistic view need not be taken as something stronger than a certain methodological commitment to a kind of explanation. As Brandon has put it, the question: 'what is a mechanism?'

\footnotetext{
has no general metaphysical answer, because the business of science is the discovery of mechanisms; so we cannot delimit in any a priori manner the mechanisms of nature. [...] The best we can do is to give an open-ended answer: a mechanism is any describable causal process $(1990,185$, emphasis added).
}

This can be generalised as follows: concepts such as mechanism, that are central in scientific practice, should be viewed as methodological postulates rather than as presupposing robust metaphysical commitments. But methodological postulates should be 'open-ended'; otherwise they would unnecessarily limit research. Far from being a trivial commitment, MM is flexible enough to foster searching for mechanisms, whatever the ontic signature of the world might be.

\section{Newton Revisited}

How does all this get connected to Newton's critique of the Old Mechanism? In a letter to Leibniz dated 16 October 1693, Newton challenged him to offer a mechanical explanation of "gravity along with all its laws by the action of some subtle matter" and to show "that the motion of planets and comets will not be disturbed by this matter". If this were available, Newton said, he would be "far from objecting". But no such explanation was forthcoming and Newton was happy to re-iterate his view that

since all phenomena of the heavens and of the sea follow precisely, so far as I am aware, from nothing but gravity acting in accordance with the laws described by me; and since nature is very simple [...] all other causes are to be rejected (Newton 2004, 108-109).

Newton does not simply say that causal explanation might not be mechanical. His point is that causal explanation should be liberated from mechanism. It'd not be enough to offer a mechanical account of the cause of gravity; the laws that gravity obeys should be mechanically explicable and, as Newton repeatedly stressed, this was not forthcoming. Though causal 
explanation matters, it doesn't matter if it is subject to various (old) mechanical constraints.

We noted already that the new mechanical conception of nature is far from the seventeenth century conception that everything should be accounted for in terms of (configurations of) matter in motion. So it's far from us to tar New Mechanism with the same brush as Old Mechanism. For instance, the key ontology of the old mechanical picture was justified, by and large a priori, whereas the key ontology of New Mechanism is grounded in scientific practice; in this case, it is practice that constrains metaphysics. Be that as it may, we are now going to argue that there exists a kind of Newtonian move against New Mechanism too.

What is clear from the present discussion is that, regardless of the main difference noted above, the new idea of mechanism is no less metaphysically loaded than the old one. Where the seventeenth century mechanists looked for stable arrangements of matter in motion subject to laws, the twenty-first century mechanists look for stable arrangements of powerful entities engaged in various activities and interactions. These mechanisms are supposed to be the building blocks of nature and the scientific task is to unravel them. They underpin "mechanistic explanations" which, as Glennan put it, show "how the organized activities and interactions of some set of entities cause and constitute the phenomenon to be explained" $(2017,223)$. Mechanistic explanation "always involves characterizing the activities and interactions of a mechanism's parts" $(2017,223)$. Where the seventeenth century mechanists saw 'action by contact' as a requisite for a proper mechanical explanation, new mechanists see powers and 'activities'.

Why is Newton's key thought relevant to the modern debates about mechanisms? The key thought, to repeat, was that causal explanation should identify causes and the laws that govern their action irrespective of whether or not these causes can be taken to satisfy further (mostly metaphysically driven) constraints. In other words, Newton showed that certain causal explanations of phenomena (in terms of non-mechanical forces) are both legitimate and complete insofar as they identify the right causes and are empirically grounded.

We take it that the point MM stresses, is, mutatis mutandis, analogous to Newton's. The point of MM is that causal explanation need not be mechanistic in the new mechanists' ontic sense, and that being couched in the way new mechanists propose, causal explanation is subjected to constraints unwarranted by scientific practice. Insofar as mechanism is a concept-in-use in science, it may well be seen referring to the causal pathway of the phenomenon to be explained, couched in the language of theories. Preserving the spirit of Newton's key thought, we might say that causal explanation is legitimate even if we bracket the issue of "what mechanisms or causes are as things in the world" (Glennan 2017,12 ) or the issue of what activities are and how they are related to powers and the like. The issue then is not "an ontological characterization of what mechanisms are as things in the world" $(2017,19)$, but a methodological characterisation of them as causal pathways described in the language of theories.

To press the analogy a bit more, questions such as: compounded? Where does one entity or activity or mechanism end, and when does 
another begin? And on what account do we decide that a collection of interacting entities is to count as a whole mechanism? (Glennan 2017, 29)

are pretty much like the questions concerning the cause of the properties of gravity that Newton thought need not be asked and answered for a scientifically legitimate conception of causal explanation.

We don't want to claim that questions such as the above are not connected to scientific practice. After all, even the question of the cause of gravity that Newton refrained from answering, was connected to scientific practice. The point, rather, we take from Newton is that answering these questions is not required for offering adequate causal explanations of the phenomena under study. Similarly,, for MM, answering questions such as the above is not required in order to have legitimate mechanistic explanations. In other words, the properties of mechanism over and above those that are required by its methodological use need not be specified; nor is there an explanatory lacuna if they are not.

According to MM, the concept of mechanism as used in practice need not, and should not, be understood in a metaphysically inflated sense. Hence, new mechanists, in offering such metaphysically inflated accounts, need to show that such accounts are indeed indispensable for doing good mechanistic science.

To conclude, as Newton remained agnostic about the underlying mechanism of gravity, so MM remains agnostic about the metaphysical ground of any particular causal pathway. As in the case of gravity, it is enough that mechanisms qua causal pathways really exist and act as they do.

\section{Acknowledgement}

An earlier version of this paper was presented at the AIPS Conference on Mechanistic Explanations, in Dortmund, 27-30 October 2016. We would like to thank the audience for comments and suggestions. Special thanks are due to Brigitte Falkenburg and Gregor Schiemann for their invitation, encouragement and comments. Many thanks to Stuart Glennan for useful comments on an earlier draft.

\section{References}

Bechtel, W. and A. Abrahamsen (2005) 'Explanation: A Mechanistic Alternative', Studies in History and Philosophy of Biological and Biomedical Sciences 36: 421-441.

Boyle, R. (1991) Selected Philosophical Papers of Robert Boyle [Stewart, M. A. (ed)], Indianapolis \& Cambridge: Hackett Publishing Company.

Brandon, R. N. (1984) 'Grene on Mechanism and Reductionism: More Than Just a Side Issue', PSA: Proceedings of the Biennial Meeting of the Philosophy of Science Association, Vol. 1984, 2: 345-353.

Brandon, R. N. (1990) Adaptation and Environment, Princeton University Press. Craver, C. F. (2013) 'Functions and Mechanisms: A Perspectivalist View' in P. Huneman (ed) Functions: Selection and Mechanisms, Dordrecht: Springer, 133-158.

Descartes, R. (1982) Principles of Philosophy [Miller, V.R. and Miller, R.P. (trans)], Dordrecht: D. Reidel Publishing Company. 
Descartes, R. (2004) René Descartes: The World and Other Writings [Gaukroger, S. (ed)], Cambridge University Press.

Dowe, P. (2000) Physical Causation, Cambridge: Cambridge University Press.

Garson, J. (2018) 'Mechanisms, Phenomena, and Functions' in Glennan S. and P.

M. Illari (eds) The Routledge Handbook of Mechanisms and Mechanical

Philosophy, New York: Routledge, 104-115.

Gillies, D. (2017) 'Mechanisms in Medicine', Axiomathes 27: 621-634.

Glennan, S. (1996) 'Mechanisms and The Nature of Causation', Erkenntnis 44: 4971.

Glennan, S. (2002) 'Rethinking Mechanistic Explanation', Philosophy of Science 69: S342-S353.

Glennan, S. (2017) The New Mechanical Philosophy, Oxford: Oxford University Press.

Harré, R. (1970) The Principles of Scientific Thinking, London: Macmillan.

Huygens, C. (1997) [1690] Discourse on the Cause of Gravity [Karen Bailey (trans)], Mimeographed.

Illari, P. M. and J. Williamson (2012) 'What is a Mechanism? Thinking about Mechanisms across the Sciences', European Journal of Philosophy of Science 2: 119-135.

Ioannidis, S. and S. Psillos (2017) 'In Defense of Methodological Mechanism: The Case of Apoptosis', Axiomathes 27: 601-619.

Ioannidis S. and S. Psillos (2018) 'Mechanisms, Counterfactuals and Laws' in Glennan S. and P. M. Illari (eds) The Routledge Handbook of Mechanisms and Mechanical Philosophy, New York: Routledge, 144-156.

Kaiser, M. I. (2018) 'The Components and Boundaries of Mechanisms' in Glennan S. and P. M. Illari (eds) The Routledge Handbook of Mechanisms and Mechanical Philosophy, New York: Routledge, 116-130.

Lakhani, S., Dilly, S. and Finlayson, C. (2009) Basic Pathology: An Introduction to the Mechanisms of Disease (4th ed), Hodder Arnold.

Leibniz, G. W. (1989) G. W. Leibniz: Philosophical Essays [Ariew, R. and Garber, D. (eds)], Indianapolis \& Cambridge: Hackett Publishing Company.

Machamer, P., L. Darden and C. F. Craver (2000) 'Thinking About Mechanisms', Philosophy of Science 67: 1-25.

Mackie, J. L. (1974) The Cement of the Universe, Oxford: Clarendon Press.

Matthews, L. J. and J. Tabery (2018) 'Mechanisms and the metaphysics of causation' in Glennan S. and P. M. Illari (eds) The Routledge Handbook of Mechanisms and Mechanical Philosophy, New York: Routledge, 131-143.

Newton, I. (2004) Philosophical Writings [Janiak, A. (ed)], Cambridge: Cambridge University Press.

Povich, M. and C. F. Craver (2018) 'Mechanistic Levels, Reduction and Emergence' in Glennan S. and P. M. Illari (eds) The Routledge Handbook of Mechanisms and Mechanical Philosophy, New York: Routledge, 185-197.

Psillos, S. (2002) Causation and Explanation, Acumen \& McGill-Queens University Press.

Psillos, S. (2011) 'The Idea of Mechanism' in P. M. Illari, F. Russo \& J. Williamson (eds) Causality in the Sciences, Oxford University Press, pp.771-788.

Salmon, W. (1997) 'Causality and Explanation: A Reply to Two Critiques', Philosophy of Science 64: 461-77.

Wilson, M. D. (1999) Ideas and Mechanism: Essays on Early Modern Philosophy, 
Princeton: Princeton University Press.

Woodger, J. H. (1929) Biological Principles: A Critical Study, London: Routledge \& Kegan Paul Ltd. 